\title{
Detection and Stabilization of a Previously Unknown 2D (Pseudo)polymorph using Lateral Nanoconfinement
}

\author{
Ana M. Bragança ${ }^{\dagger \#, ~ A n d r e a ~ M i n o i a ~}{ }^{\ddagger \#}$, Roelof Steeno ${ }^{\dagger}$ Johannes Seibel ${ }^{\dagger}$, Brandon E. Hirsch ${ }^{\dagger}$ Lander \\ Verstraete ${ }^{\dagger}$, Oleksandr Ivasenko ${ }^{\dagger}$ Klaus Müllen,${ }^{\perp}$ Kunal S. Mali+*, Roberto Lazzaroni ${ }^{* *}$, and Steven De \\ Feyter+*
}

†Division of Molecular Imaging and Photonics, Department of Chemistry, KU Leuven, Celestijnenlaan 200F, 3001 Leuven, Belgium, $\neq$ Laboratory for Chemistry of Novel Materials, Materials Research Institute, University of Mons, Place du Parc 20, 7000 Mons, Belgium, ${ }^{\perp}$ Max Planck Institute for Polymer Research, Ackermannweg 10, D-55128 Mainz, Germany

\begin{abstract}
:
We report on the detection and stabilization of a previously unknown 2D pseudopolymorph of an alkoxy isophthalic acid using lateral nanoconfinement. The self-assembled molecular networks formed by the isophthalic acid derivative were studied at the interface between covalently modified graphite and an organic solvent. When self-assembled on graphite with moderate surface coverage of covalently bound aryl groups, a previously unknown metastable pseudopolymorph was detected. This pseudopolymorph, which was presumably 'trapped' in between the surface bound aryl groups, underwent a timedependent phase transition to the stable polymorph typically observed on pristine graphite. The stabilization of the pseudopolymorph was then achieved by using an alternative nanoconfinement strategy where the domains of the pseudopolymorph could be formed and stabilized by restricting the self-assembly in nanometer-sized shallow compartments produced by STM-based nanolithography carried out on graphite surface carrying a high density of covalently bound aryl groups. These experimental results are supported by molecular mechanics and molecular dynamics simulations which not only provide important insight into the relative stabilities of the different structures but also shed light onto the mechanism of the formation and stabilization of the pseudopolymorph under nanoscopic lateral confinement.
\end{abstract}




\section{Introduction}

Polymorphism, defined as the ability of a solid material to adopt two or more crystalline forms, may lead to distinct physical and/or chemical properties while maintaining the same chemical composition. ${ }^{1} \mathrm{~A}$ rational control over the formation of polymorphs, and thus their functional properties, is highly desirable in several technological sectors (pharmaceutical, biomineral, food and explosive). ${ }^{2}$ Numerous approaches have been developed to control nucleation and crystallization pathways. In solution, nonphotochemical light-induced nucleation ${ }^{3}$ or crystallization in microemulsions ${ }^{4}$ were used to control the formation of different polymorphs of glycine or mefenamic acid, respectively. In the late 1990's, Ward and others used engineered nanoporous matrices like porous glass or block copolymer monoliths to study crystallization under confinement to control polymorph formation.5-7 On solid surfaces, thiolbased monolayers on gold or silane-based self-assembled monolayers (SAMs) have been used as chemisorbed templates for controlled crystal nucleation and 3D growth through specific interfacial interactions that help regulate polymorph selection. ${ }^{8}$

Polymorphism is not exclusive to 3D materials. Structural polymorphism has also been observed and investigated for two-dimensional self-assembled molecular networks (2D-SAMN) physisorbed on atomically flat and conductive solid substrates. Scanning tunneling microscopy (STM) is an effective imaging tool to probe structural polymorphism with sub-molecular resolution both under ultrahigh vacuum conditions (UHV) and at the liquid-solid interface.9-12 For some systems investigated under UHV conditions, different polymorphs have been isolated on small terraces confined between substrate step edges. This was attributed to the effect of confinement and their impact on the balance between the different forces governing the self-assembly on surfaces: molecule-molecule, molecule-substrate and molecule-step edge interactions.13-15 One of the early and rather ingenious attempts to study selfassembly under confinement consisted of compartmentalizing self-assembly in flat-bottomed, circular pits grown on HOPG. ${ }^{16-18}$ The pits were made by chemical modification of HOPG by heating it in the presence of $\mathrm{O}_{2}$ at $650^{\circ} \mathrm{C}$. These so called 'molecule corrals' were used to study isolated populations of molecules in corrals and on surrounding terraces. These early studies revealed that nucleation events occurring on the terraces are independent of those in the pits and the nucleation rate depends on the pit size. ${ }^{19}$ Controlling the size, shape and orientation of such pits however, is not straightforward and calls for a robust and reproducible method for creating such nanoconfined spaces. On large terraces, where the self-assembly occurs without any lateral confinement, a variety of factors such as concentration $20-23$, temperature ${ }^{24-27}$, solvent ${ }^{28-30}$, nature of the substrate ${ }^{31-33}$, influence of the bias applied to the substrate ${ }^{34-38}$ or the application of shear-flow ${ }^{39}$ were shown to influence the (pseudo)polymorph appearance at the solid-liquid interface.

Polymorphism is also closely related to the formation of fundamentally different crystalline structures both in 2D and 3D where the solvent molecules co-crystallize together with the molecule of interest into a crystalline lattice.29, 40-41 Such systems are referred to as pseudopolymorphs, solvates or a subclass of cocrystals, though the precise terminology has been a subject of debate. ${ }^{42}$ The existence of 
such pseudopolymorphs, the term we will use in this manuscript, is of particular importance in pharmaceutical sciences.

Here, we present two strategies to create nanoscale spatial confinement with the aim to control the assembly process at the molecular level and to generate and stabilize (select) polymorphs or pseudopolymorphs (Figure 1c), at the liquid-solid interface, the solid being highly oriented pyrolytic graphite (HOPG). The first approach towards nanoconfinement is based on covalently attaching aryl groups to the basal plane of graphite with controlled surface coverage (Figure 1a), creating a chemically modified HOPG surface (CM-HOPG, Figure 1e) with moderate density of aryl groups. ${ }^{43-44}$ In the second
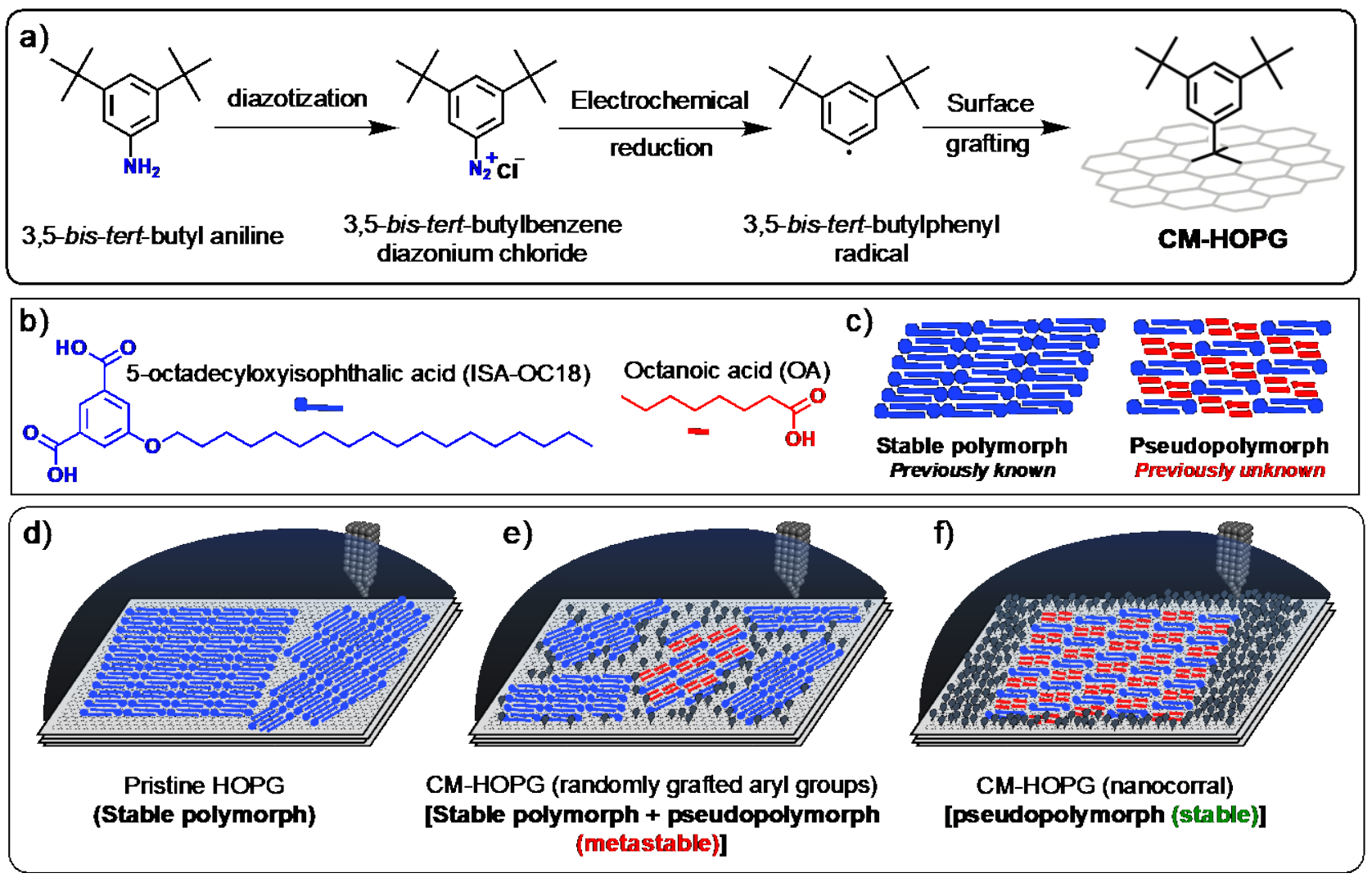

Figure 1. (a) Reaction scheme showing the functionalization protocol used for attaching aryl groups to the surface of graphite to yield covalently modified graphite (CM-HOPG). (b) Molecular structures and schematic representations of 5-octadecyloxyisophthalic acid (ISA-OC18, blue) and octanoic acid (OA, red). (c) Schematic representation of the stable, previously known ISA-OC18 polymorph, and a previously unknown pseudopolymorph detected under nanoconfinement in this work. (d-f) Schematics showing different outcomes of the self-assembly process on pristine (d), and CM-HOPG (e, f) substrates. When assembled at the 1-octanoic acid/HOPG interface, ISA-0C18 assembles exclusively into the wellknown stable polymorph as depicted in (d). When the assembly takes place on CM-HOPG with an intermediate density of chemisorbed aryl units, both polymorphs are formed, however the pseudopolymorph was found to be metastable (e). When compartmentalized into well-defined shallow nanocorrals produced in the presence of ISA-0C18 solution, the otherwise metastable pseudopolymorph can be obtained almost exclusively and remains stable. 
approach, shallow nanocompartments with well-defined size and shape were created using STM based nanolithography carried out on CM-HOPG with high density of aryl groups (Figure 1f). ${ }^{45}$ Scanning probe nanolithography has been previously used for nanofabrication and simultaneous self-assembly of thiols chemisorbed on Au. ${ }^{46-48}$ We demonstrate that the aforementioned approaches could be used for the (plausible screening), detection and stabilization of previously unknown (pseudo)polymorphs for a physisorbed molecular system (Figure 1d-f) that has been investigated for more than 20 years, namely 5-octadecyloxy-isophthalic acid (ISA-OC18). The experimental results have been aptly supported by molecular mechanics (MM) and molecular dynamics (MD) simulations, which not only provide important insight into the relative stabilization energies of the structures involved but also reveal a plausible pathway that leads to the formation and the stabilization of the pseudopolymorph under nanoconfinement.

\section{Results and Discussion}

The self-assembly of ISA-OC18 (Figure 1b) at the solid-liquid interface has been extensively studied in the past. ${ }^{49-53}$ As displayed in the STM image presented in Figure 2a, ISA-0C18 commonly assembles into a lamellar structure with the ISA head groups (bright features) arranged in a head-to-head orientation, while the alkyl chains (striped dark features) lie flat along one of the major symmetry axes of the graphite lattice. The alkyl chains of the molecules in adjacent lamellae interdigitate (Figure 2a,c). This close-packed arrangement is stabilized by van der Waals interactions between the interdigitated alkyl chains and the tetrameric hydrogen bonding ${ }^{10}$ between the ISA head groups ( also see Figure S1, S2 in the supporting information). This structure is referred to as 'the stable polymorph' hereon.

When assembled at the interface between $\mathrm{OA}$ and $\mathrm{CM}-\mathrm{HOPG}$ at relatively high solute concentrations (0.1-0.2 mM), ISA-0C18 was found to assemble into a completely new structure (Figure $2 \mathrm{~b})$ alongside the stable polymorph. This new structure is referred to as 'the pseudopolymorph' hereon. Similar to our previous work, ${ }^{43-45}$ CM-HOPG was obtained by covalently binding individual 3,5-bis-tertbutylphenyl (3,5-TBP) groups to the pristine graphite surface (300 - 475 units per $\left.100 \times 100 \mathrm{~nm}^{2}\right)$ using electrochemical grafting of the corresponding diazonium salt (see Figure 1a). Here onwards, we refer to these covalently bound 3,5-TBP groups as 'the defects'. The number of such defects per unit area can be readily controlled by careful adjustment of the experimental conditions (see more details in SI, Section 1).

The high-resolution STM image of the pseudopolymorph shown in Figure 2b shows alternating bright features that appear as a collection of three dots. We attribute these features to the phenyl groups of the ISA units arranged in a zig-zag configuration. In between the zig-zag pattern, striped features with two sets of lengths can be visualized. We assign the longer features to the octadecyloxy chains of the ISA-0C18 and the shorter ones to the co-adsorbed solvent molecules. Figure $2 \mathrm{~d}$ shows a molecular model for the pseudopolymorph which was built using the experimentally obtained unit cell. As evident 

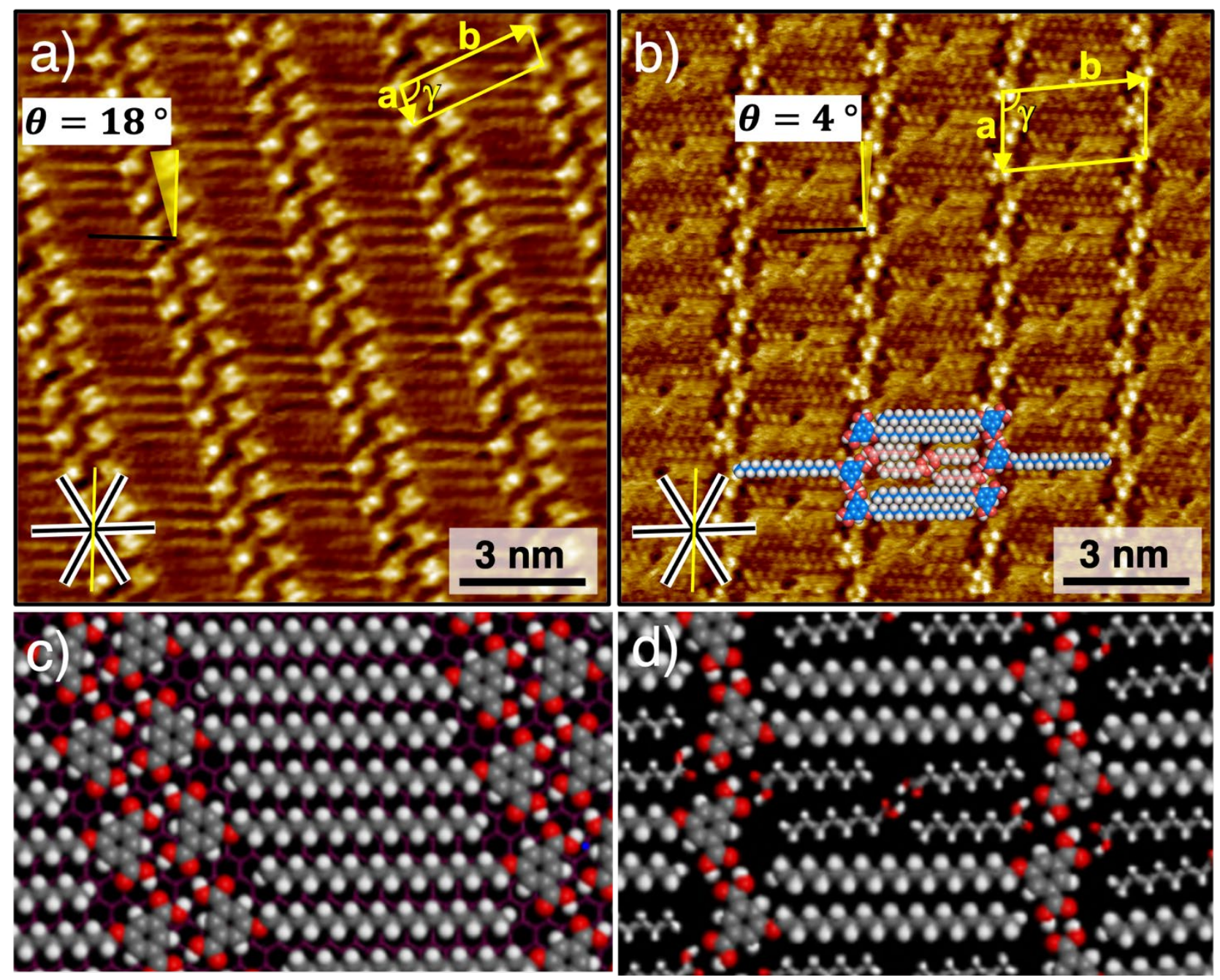

Figure 2. High resolution STM images of the stable polymorph (a) and the pseudopolymorph (b). The stable polymorph forms an azimuthal angle of $\pm 18^{\circ}$ with respect to the direction perpendicular to the main symmetry axes, while the pseudopolymorph is defined by an angle of $\pm 4^{\circ}$. The unit cells are indicated in yellow. Stable polymorph: $\mathrm{a}=1.0 \pm 0.1 \mathrm{~nm}, \mathrm{~b}=3.7 \pm 0.1 \mathrm{~nm}, \gamma=98.7 \pm 1.1^{\circ}$ and pseudopolymorph: $\mathrm{a}=1.9 \pm 0.1 \mathrm{~nm}, \mathrm{~b}=3.2 \pm 0.1 \mathrm{~nm}$ and $\gamma=97.5 \pm 1.3^{\circ}$. Imaging parameters: $(\mathrm{a}) \mathrm{V}_{\text {bias }}=-$ $0.65 \mathrm{~V}, \mathrm{I}_{\text {set }}=80 \mathrm{pA}$; (b) $\mathrm{V}_{\text {bias }}=-0.80 \mathrm{~V}, \mathrm{I}_{\text {set }}=80 \mathrm{pA}$. (c, d) Molecular mechanics optimized models depicting the organization of molecules in the stable polymorph (c) and the pseudopolymorph (d).

from the model, the network comprises a periodic arrangement of ISA-OC18 and OA molecules coadsorbed in a 1:2 ratio. Unlike the stable polymorph, where the ISA-OC18 molecules assemble via tetrameric hydrogen bonding, the pseudopolymorph consists of cyclic homomeric (OA …OA, ISA-

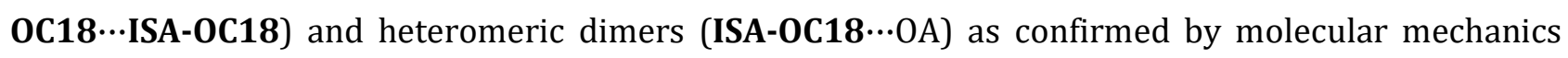
simulations (Figure S3 in the supporting information). The strong directionality of cyclic dimerization forces the pseudopolymorph to sacrifice close packing to improve the geometry of these interactions. ${ }^{54}$ 
Table 1. Unit cell parameters and stabilization energies of the (pseudo)polymorphs of ISA-0C18 formed at the solution-solid interface. The details of $\mathrm{E}_{\text {ISA }}$ calculations are provided in the supporting information.

\begin{tabular}{|c|c|c|c|c|c|c|c|}
\hline & \multicolumn{3}{|c|}{ Unit cell (experimental) } & \multicolumn{3}{|c|}{ Unit cell (calculated) } & \multirow{2}{*}{$\begin{array}{c}\mathrm{E}_{\mathrm{ISA}} \\
(\mathrm{kcal} / \mathrm{mol})\end{array}$} \\
\hline & $\mathrm{a}(\mathrm{nm})$ & $\mathrm{b}(\mathrm{nm})$ & $\gamma\left({ }^{\circ}\right)$ & $\mathrm{a}(\mathrm{nm})$ & $\mathrm{b}(\mathrm{nm})$ & $\gamma\left({ }^{\circ}\right)$ & \\
\hline Stable polymorph & $1.0 \pm 0.1$ & $3.7 \pm 0.1$ & $98.7 \pm 1.1$ & 0.94 & 3.8 & 96.6 & 1283 \\
\hline Pseudopolymorph & $1.9 \pm 0.1$ & $3.2 \pm 0.1$ & $97.5 \pm 1.3$ & 1.9 & 3.2 & 94.6 & 1689 \\
\hline
\end{tabular}

The pseudopolymorph however, was found only sporadically on the surface of CM-HOPG which carries randomly distributed surface defects. Its occurrence was found to depend on the local distribution of chemisorbed 3,5-TBP units and the size, shape and proximity of the stable polymorph (see Figures S4 and S5 in the supporting information). Furthermore, under typical experimental conditions, the pseudopolymorph was found to be metastable and slowly transitioned into the stable polymorph with time. Typically, the pseudopolymorph is present in the early stages of the experiment as evident from sequential STM images recorded immediately after drop casting ISA-0C18 solution on the surface of CM-HOPG (Figure S4 in the supporting information).

Figure 3 shows a set of sequential STM images where the gradual transition of the pseudopolymorph into the stable polymorph was followed over approximately 13 minutes. It can be readily noticed that the domains of the pseudopolymorph (red, Figure 3a) gradually transition into those of the stable polymorph (blue, Figure 3b-3d) with time. In general, it was observed that the pseudopolymorph survives for longer duration only when it is isolated or sits adjacent to a smaller domain of the stable polymorph (Figure S5 in the supporting information). We note that, the pseudopolymorph was never detected on pristine HOPG within the range of concentration studied here $(0.2 \mathrm{mM}$ to $57.5 \mu \mathrm{M})$, not even at concentrations at which only sub-monolayer coverage (38 $\mu \mathrm{M})$ was found (see Figure S6 and S7 in the supporting information) The pseudopolymorph however could be transiently formed and observed after disruption of the stable polymorph by application of several voltage pulses to the STM tip. The pulsing disrupts the domain of the stable polymorph and the pseudopolymorph could be imaged briefly (Figure S8 in the supporting information). These combined observations indicate that the pseudopolymorph is a kinetic structure that is plausibly formed first and gets converted into the thermodynamically more stable polymorph as a function of time.

A mixed MD/MM approach, called MD/Quench iterative method (see experimental section in the supporting information for details), was used to investigate the most stable structure for the two networks, and to compare their relative stability. For each structure, three possible conformations were considered for the ISA-OC18 carboxylic groups, thus producing three possible monolayers with a 


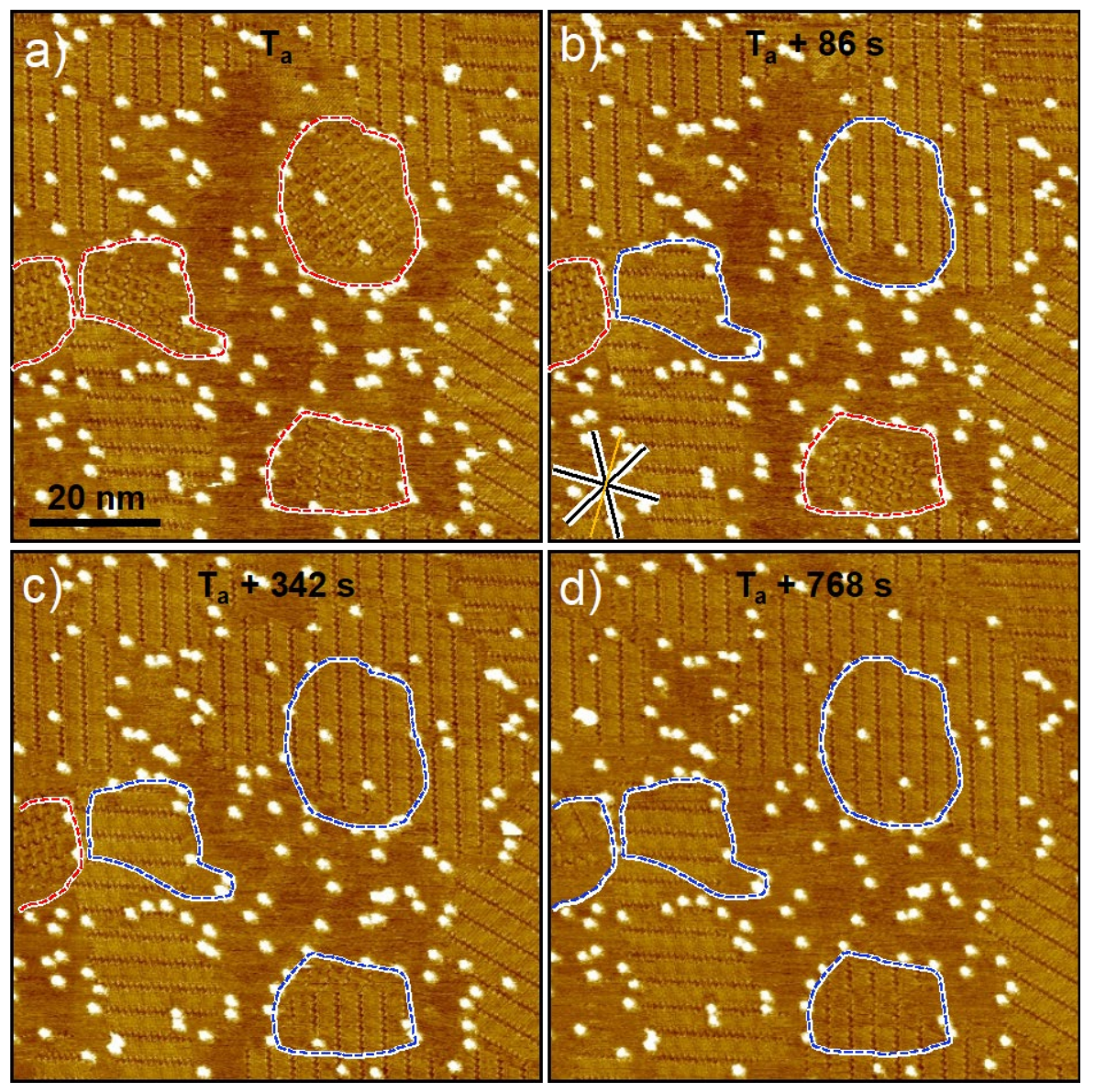

--- pseudopolymorph ---stable polymorph

Figure 3. Sequential STM images showing a gradual transition from the metastable pseudopolymorph (in red) to the stable polymorph (in blue) over a period of 13 minutes. Imaging parameters: (a)-(d) $V_{\text {bias }}$ $=-0.70 \mathrm{~V}, I_{\text {set }}=80 \mathrm{pA}$. The bright dots are the chemisorbed 3,5-TBP units.

different type of intermolecular H-bonding network. For the stable polymorph, the unit cell parameters of all three possible assemblies match closely with that of the experimentally measured unit cell. (see Table S1); however, for only one specific conformation ('Head 3'; see Figure S1 in the supporting information), the orientation of the monolayer with respect to graphite symmetry axes was found to be in good agreement with the experimental data.

For the pseudopolymorph, two patterns were examined (see Figure S3 in the supporting

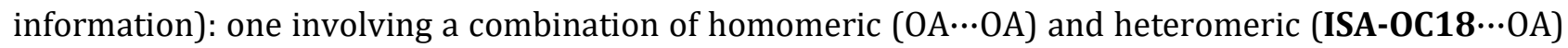
H-bonding and the other stabilized by only homomeric H-bonds (OA $\cdots$ OA, ISA-OC18 $\cdots$ ISA-OC18). In the latter case, the solvent molecules simply fill up the space in between the ISA-0C18 molecules without specifically binding to them. The former organization was found to be more stable than the latter. More importantly, the potential energies extracted from the theoretical models associated to the stability of the two different ISA-networks found in the two polymorphs clearly show that the known polymorph is systematically more stable than the pseudopolymorph (Table 1). 
Even though the use of CM-HOPG with random distribution of aryl groups provided a way to influence structural polymorphism, the sporadic occurrence and the time dependent transition of the pseudopolymorph made it difficult to predict its occurrence or characterize its structure in detail. These experiments essentially suggested that to extend the lifetime of the pseudopolymorph, it should be spatially isolated from the stable polymorph to prevent its removal from the surface.

Thus, an alternative, more calibrated approach that involves the use of geometrically welldefined, isolated nanometer sized compartments to study self-assembly was employed. These nanocompartments, hereon referred to as corrals, were created using STM-based nanoshaving of CMHOPG carrying a high density of grafted units. ${ }^{45,55}$ In a typical nanoshaving experiment, the covalently bound 3,5-TBP units are precisely detached from the surface by scanning the STM tip close to the surface at higher tunneling currents. We have previously demonstrated that such nanoshaving and the subsequent detachment of the aryl groups proceeds with restoration of the $\mathrm{sp}^{2}$ hybridisation of the graphite lattice..$^{43}$ This nanoshaving can be either carried out ex-situ in air, in the absence of the selfassembling molecules or in-situ, at the liquid-solid interface, in the presence of the solution of the assembling molecules. It follows that, in the in-situ process, the physisorption and the self-assembly of molecules occurs in concert with the nanoshaving as the surface of pristine graphite becomes available with each scanned line (Figure S9 in the supporting information). On the other hand, when the solution of the assembling molecules is drop casted onto the corrals formed ex-situ on CM-HOPG, the molecules are exposed to pristine surface at once, akin to that on the surface of pristine unmodified graphite (see Figure 4).
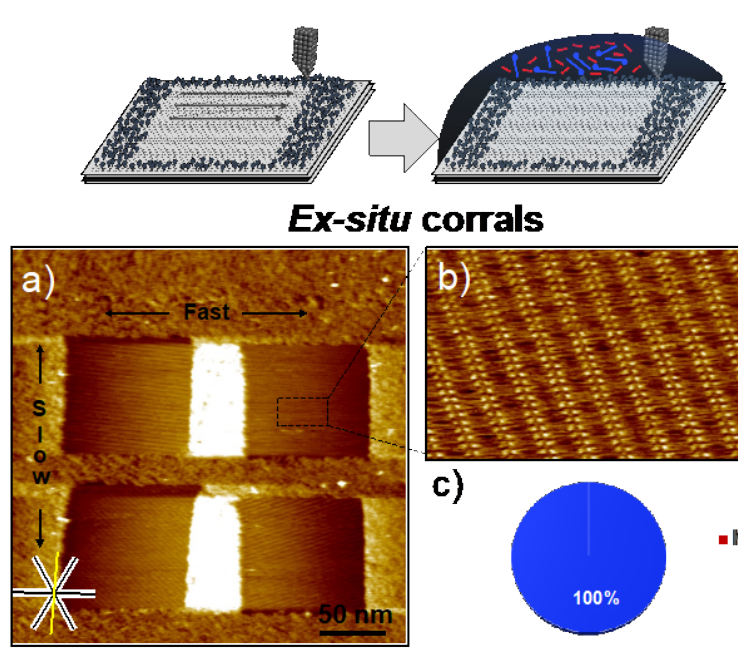
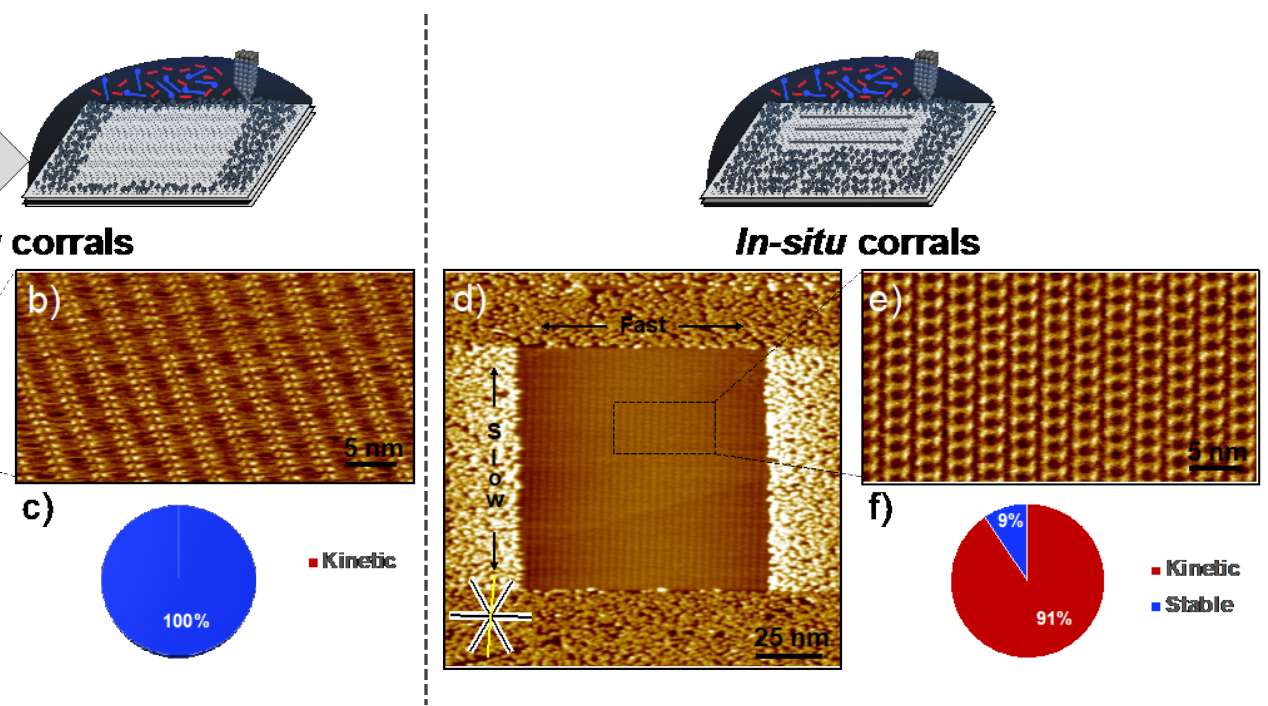

Figure 4. STM images showing the assembly of ISA-OC $\mathbf{C O}_{\mathbf{1 8}}(56 \mu \mathrm{M})$ in corrals formed ex-situ (a), and insitu (b). The size of the nanocorral is approximately $90 \mathrm{~nm} \times 90 \mathrm{~nm}$. Fast and slow nanoshaving directions are indicated in the image. When corrals are fabricated ex-situ only the stable polymorph is detected (a-c). However, when corrals are fabricated in-situ in the presence of the ISA-0C $\mathbf{1 8}_{\mathbf{8}}$ solution, the pseudopolymorph is detected $91 \%$ of the times (d-f). 
Figure 4 presents representative STM data showing the comparison of the outcome of the two types nanoshaving experiments. When the nanoshaving and the self-assembly are decoupled from each other (the ex-situ process), the corrals were filled exclusively with the stable polymorph (Figure 4a-c). However, the result was found to be dramatically different in the case of the in-situ nanoshaving experiment carried out in the presence of ISA-0C18 solution (0.1-0.2 mM) where the occurrence of pseudopolymorph inside corrals increased up to 50\%. Furthermore, the pseudopolymorph can be formed predominantly in the in-situ fabricated corrals by simply lowering the concentration of ISAOC18, in an in-situ nanoshaving experiment. Thus, the pseudopolymorph was detected in $91 \%$ of corrals (total of 53 corrals examined) when the solution concentration was lowered to $56 \mu \mathrm{M}$ (Figure $4 \mathrm{~d}-\mathrm{f}$ ). More importantly, the pseudopolymorph remained unperturbed upon confinement even after continuous scanning for long periods of time ( $>1 \mathrm{~h}$, Figure S11 in the supporting information). It remained stable until intentionally perturbed by a large voltage pulse, in which case the corrals were gradually filled up by the stable polymorph (Figure S12 in the supporting information).

So, what do the randomly grafted CM-HOPG and in-situ nanoshaved corrals have in common that allows the formation of the pseudopolymorph? A close inspection of the STM data obtained on CM-HOPG with randomly grafted defects revealed that the domains of the pseudopolymorph exhibit a high aspect ratio with approximately a rectangular shape containing multiple adjacent lamellae rather than a few long lamellae (Figure S9a in the supporting information). Similar shape is created during the initial stages of the in-situ corral formation when the molecular self-assembly occurs. Thus, we reason that the gradual exposure of the pristine HOPG surface in the presence of the ISA-0C18 solution must play a major role in the stabilization of the pseudopolymorph. Indeed, statistical analysis revealed that in $85 \%$ of the corrals the alkyls chains are aligned with the fast-shaving direction (Figure S13 in the supporting information), supporting the idea that the domain growth occurs while the corral is being formed. Continuous nanoshaving allows the domain to grow until the corral is fully filled. If this hypothesis is valid, one should observe a preference for the occurrence of the pseudopolymorph if the nanocorrals are formed (in-situ) in the shape of a downward facing triangle compared to those shaved in the form of an upward facing triangle. This is because, for the downward facing triangle, the nanoshaving starts at the base of the triangle which creates the same type of high aspect ratio pristine area as that created in the case of the rectangular or square corral. On the other hand, for the latter case, the nanoshaving starts at the apex creating low aspect ratio triangular areas thus disfavoring the formation of the pseudopolymorph. Experimental results presented in Figure S14 of the supporting information indeed suggest that for the downward facing triangles, the pseudopolymorph was detected in $89 \%$ of the corrals compared to only $56 \%$ in the case of the upward facing triangles. Based on these observations, we suggest a possible mechanism. The size of the nanocorrals as such does not have a strong influence on the stabilization of the pseudopolymorph. Results described in Figure S15 of the supporting information clearly indicate that pseudopolymorph can be stabilized in larger corrals $\left(200 \times 120 \mathrm{~nm}^{2}\right)$ and that the high aspect ratio thin channels created in the initial stages of nanoshaving is the 
determining factor for polymorph selection rather than the actual corral size. Furthermore, nanoshaving on a domain of the stable polymorph physisorbed on pristine surface does not yield the pseudopolymorph (Figure S16) highlighting the importance of the chemisorbed organic layer for the nanoshaving experiments performed in this work.

Given that the solvent is always present in large excess in the solution and also given the fact that OA itself is capable of forming a stable self-assembled network on the HOPG surface, ${ }^{56}$ we reason that, at least locally, the interaction of solvent molecules with the substrate and their initial on-surface organization is possibly the first step in the self-assembly process. MM/MD simulations show that OA physisorbs on CM-HOPG, with a clear tendency to form hydrogen bonded dimers, the alkyl chains orienting parallel to narrow trenches created by the removal of grafted molecules (in open areas, the hydrogen bonded OA dimers are found to take several orientations). This result strongly supports the view that in the initial stages of nanoshaving, OA and OA dimers, if not other alkylated species, hydrogenbonded or not, align with their alkyl chains with the fast nanoshaving direction. To mimic the early stages of self-assembly induced by the nanoshaving process, a very narrow trench (width is $\sim 1 \mathrm{~nm}$ ) consisting of graphene edges (wall height $\sim 0.7 \mathrm{~nm}$ ) was modelled to prevent diffusion of molecules outside the trench. Again, the solvent molecules, interacting by hydrogen bonding, align along the long axis of the trench (Figure $5 \mathrm{a}$, b). ISA-OC18 then adsorbs preferentially near defects in the physisorbed OA layer, thereby expelling OA molecules from the trench and aligning with its alkyl chain parallel to the long axis of the trench (Figure 5c, d). ISA-OC18 adsorption distorts and destabilizes the OA selfassembly in the trench, promoting the desorption of OA molecules and their replacement by other OA molecules or ISA-OC18. Also, other factors may contribute to the formation and stabilization of the pseudopolymorph under confinement. As the cyclic hydrogen bonds stabilizing the homomeric and the

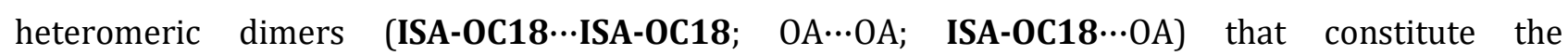
pseudopolymorph, are stronger than the catemeric hydrogen bonding motif that stabilizes the ISAoc18 molecules in stable polymorph, and involve fewer interacting species, their formation is probably faster. In addition, as such cyclic hydrogen bonds are dominant in solution for carboxylic acids, adsorption in the trenches may occur from hydrogen-bonded dimers, a favorable situation in light of the narrow width of the trenches. ${ }^{57}$ All these factors favor the fast formation of the pseudopolymorph, and the confinement protects it from fast reorganization to the more stable polymorph.

The ability to nucleate and stabilize previously unknown polymorphs using the nanoshaving approach demonstrated above is appealing, especially given the fact the pseudopolymorph is otherwise unstable and is readily removed from the surface at the gain of the stable, known polymorph. This relates to the well-known Ostwald ripening behavior routinely observed for monolayers formed at the solution-solid interface, where the domains of a stable polymorph increase in size at the expense of those of the less stable polymorph. This, however, is not the case when one considers the encapsulation of the pseudopolymorph within the nanocorrals. Once formed during the nanoshaving process, due to the lack of proximity of the stable polymorph and also possibly due to the barrier involved in the process 
of desorption, the pseudopolymorph remains stable inside the nanocorrals. The walls of the nanocorrals essentially serve as static boundaries that merely act as non-interacting container walls.

Another exciting possibility is that the nanoshaving/confinement approach may allow to verify the existence of the Ostwald's rule of stages for monolayers formed at the solution-solid interface. In fact, one can also hypothesize that the pseudopolymorph observed in the present case may in fact be the kinetic structure always formed in the early stages of self-assembly even on pristine surfaces. However, given the slow nature of the STM experiment, one often does not capture these embryonic stages. The voltage pulsing experiment, where the destruction of the stable polymorph formed on the pristine surface, led to momentary formation of the pseudopolymorph does indicate this possibility. This hypothesis, as exciting as it is, however, needs to be pursued further with rigorous experimental and theoretical treatment.
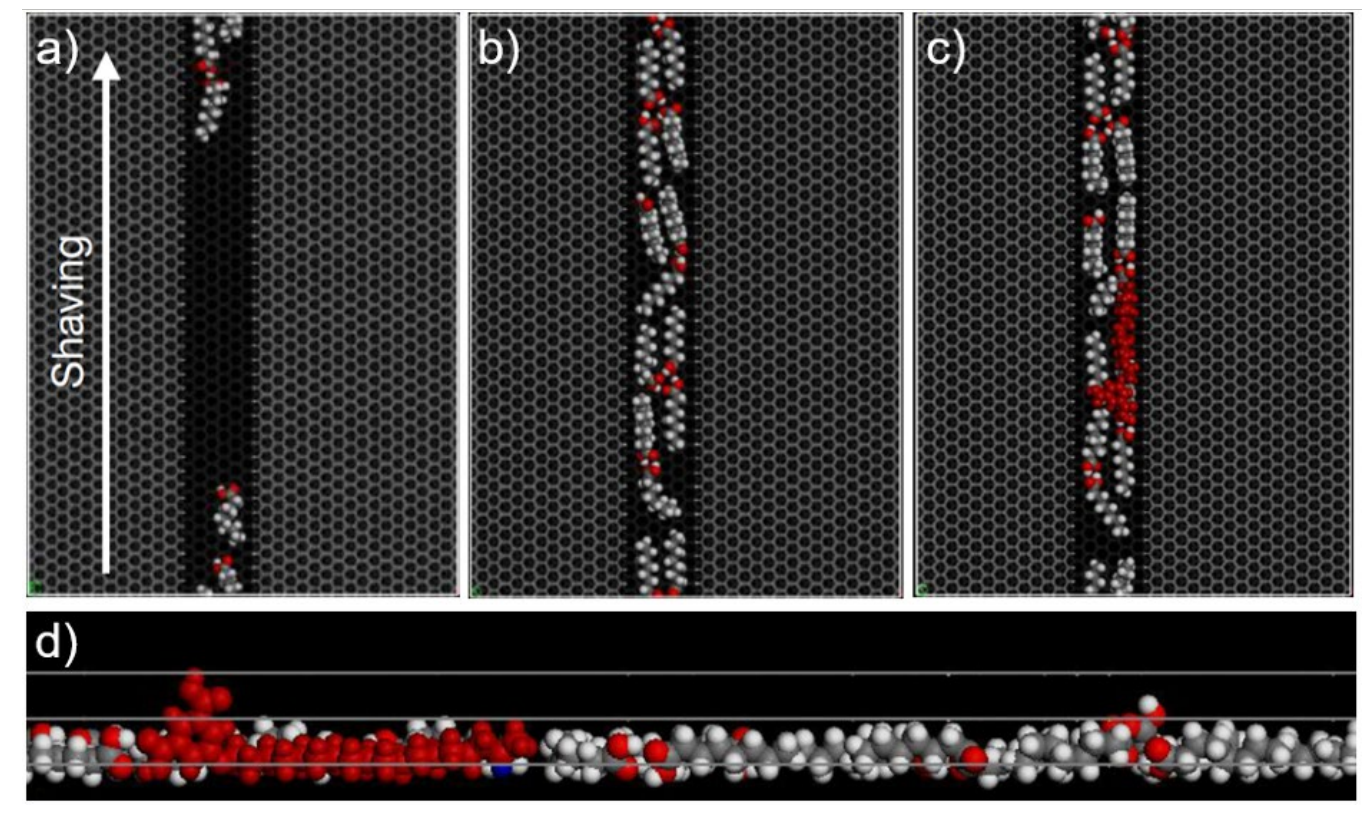

Figure 5. MM/MD modeling of the adsorption of OA and ISA-OC 18 in narrow trenches on the HOPG surface. For the sake of simplicity, the walls of the trenches simply consist of two graphene layers. (a) few OA molecules adsorbed in the trench (top view). (b) higher density arrangement of OA molecules in the trench. OA molecules interact by hydrogen bonding. (c) adsorption of ISA-OC $\mathbf{1 8}$ in a trench filled by OA molecules. (d) side view of part of (c). The physisorbed ISA-OC $\mathbf{1 8}_{\mathbf{1 8}}$ molecule is highlighted in red.

\section{Conclusions}

In the results presented above, we have successfully demonstrated that aryl groups covalently bonded to the basal plane of graphite act as kinetic barriers to the formation of 2D-SAMNs and thus affect the kinetics and thus the outcome of the self-assembly process. When using randomly grafted aryl units, this approach enabled the detection of a pseudopolymorph which was found to be metastable under the given experimental conditions. This pseudopolymorph is otherwise inaccessible on pristine surfaces. Knowing that the kinetic blockade exerted by the covalently bound aryl groups may in part transiently 
stabilize the pseudopolymorph, a more calibrated approach to nanoconfinement, namely scanning probe nanolithography on densely grafted surface allowed more reliable and predictable detection as well as stabilization of the otherwise metastable pseudopolymorph. These results clearly reveal that akin to crystallization under nanoconfinement in bulk, spatial confinement in 2D also has an effect on the stabilization of polymorphs and might even reveal the existence of previously unreported ones. Insight into a possible mechanism for the preferred formation and stabilization under nanoconfinement conditions was provided by molecular mechanics and dynamics simulations. The identification and stabilization of new phases could have practical consequences in the formation of functional surfaces.

\section{ASSOCIATED CONTENT}

Supporting Information

The Supporting Information is available free of charge at https://pubs.acs.org/doi/

Experimental materials and methods, details of the molecular mechanics and molecular dynamics simulations, additional and supporting STM data as well as supporting data from the simulations. (PDF)

\section{AUTHOR INFORMATION}

Corresponding Authors

Kunal S. Mali

Division of Molecular Imaging and Photonics, Department of Chemistry, KU Leuven, Celestijnenlaan 200F, 3001 Leuven, Belgium

Email: kunal.mali@kuleuven.be

Roberto Lazzaroni

Laboratory for Chemistry of Novel Materials, Materials Research Institute, University of Mons, Place du Parc 20, 7000 Mons, Belgium

Email: roberto.lazzaroni@umons.ac.be

Steven De Feyter

Division of Molecular Imaging and Photonics, Department of Chemistry, KU Leuven, Celestijnenlaan 200F, 3001 Leuven, Belgium

Email: steven.defeyter@kuleuven.be

\section{Author Contributions}

\#A. Bragança and A. Minoia contributed equally to this work

\section{Notes}

The authors declare no competing financial interest.

\section{Acknowledgement}


The authors also gratefully acknowledge financial support from the Fund of Scientific Research Flanders (FWO), KU Leuven - Internal Funds (C14/19/079). J.S. acknowledges financial support through a Marie Skłodowska-Curie Individual Fellowship (EU project 789865 - EnSurf). This work was in part supported by FWO and FNRS under EOS 30489208. The modelling studies are supported by FNRS (CECI, under Grant 2.5020.11) and by Wallonie (ZENOBE Tier-1 facility, grant 1117545).

\section{References:}

1. Bernstein, J.; Davey, R. J.; Henck, J.-O., Concomitant Polymorphs. Angew. Chem. Int. Ed. 1999, 38, 3440-3461.

2. Bernstein, J., Polymorphism in Molecular Crystals. Claredon Press: Oxford: 2002.

3. Garetz, B. A.; Matic, J.; Myerson, A. S., Polarization Switching of Crystal Structure in the Nonphotochemical Light-Induced Nucleation of Supersaturated Aqueous Glycine Solutions. Phys. Rev. Lett. 2002, 89, 175501.

4. Nicholson, C. E.; Chen, C.; Mendis, B.; Cooper, S. J., Stable Polymorphs Crystallized Directly under Thermodynamic Control in Three-Dimensional Nanoconfinement: A Generic Methodology. Cryst. Growth Des. 2011, 11, 363-366.

5. Hamilton, B. D.; Ha, J.-M.; Hillmyer, M. A.; Ward, M. D., Manipulating Crystal Growth and Polymorphism by Confinement in Nanoscale Crystallization Chambers. Acc. Chem. Res. 2012, 45, 414-423.

6. Beiner, M.; Rengarajan; Pankaj, S.; Enke, D.; Steinhart, M., Manipulating the Crystalline State of Pharmaceuticals by Nanoconfinement. Nano Lett. 2007, 7, 1381-1385.

7. Meldrum, F. C.; O'Shaughnessy, C., Crystallization in Confinement. Adv. Mater. 2020, 32, 2001068.

8. Singh, A.; Lee, I. S.; Kim, K.; Myerson, A. S., Crystal growth on self-assembled monolayers. Crystengcomm 2011, 13, 24-32.

9. Slater, A. G.; Perdigão, L. M. A.; Beton, P. H.; Champness, N. R., Surface-Based Supramolecular Chemistry Using Hydrogen Bonds. Acc. Chem. Res. 2014, 47, 3417-3427.

10. Ivasenko, O.; Perepichka, D. F., Mastering Fundamentals of Supramolecular Design with Carboxylic Acids. Common Lessons from X-ray Crystallography and Scanning Tunneling Microscopy. Chem. Soc. Rev. 2011, 40, 191-206.

11. Goronzy, D. P.; Ebrahimi, M.; Rosei, F.; Arramel; Fang, Y.; De Feyter, S.; Tait, S. L.; Wang, C.; Beton, P. H.; Wee, A. T. S.; Weiss, P. S.; Perepichka, D. F., Supramolecular Assemblies on Surfaces: Nanopatterning, Functionality, and Reactivity. ACS Nano 2018, 12, 7445-7481.

12. Elemans, J. A. A. W.; Lei, S.; De Feyter, S., Molecular and Supramolecular Networks on Surfaces: From Two-Dimensional Crystal Engineering to Reactivity. Angew. Chem. Int. Ed. 2009, 48, 72987332. 
13. Kühne, D.; Klappenberger, F.; Decker, R.; Schlickum, U.; Brune, H.; Klyatskaya, S.; Ruben, M.; Barth, J. V., Self-Assembly of Nanoporous Chiral Networks with Varying Symmetry from Sexiphenyl-dicarbonitrile on Ag(111). J. Phys. Chem. C 2009, 113, 17851-17859.

14. de Oteyza, D. G.; Barrena, E.; Dosch, H.; Wakayama, Y., Nanoconfinement Effects in the SelfAssembly of Diindenoperylene (DIP) on $\mathrm{Cu}(111)$ surfaces. Phys. Chem. Chem. Phys. 2009, 11, 8741-8744.

15. Fan, Q.; Dai, J.; Wang, T.; Kuttner, J.; Hilt, G.; Gottfried, J. M.; Zhu, J., Confined Synthesis of Organometallic Chains and Macrocycles by $\mathrm{Cu}-0$ Surface Templating. ACS Nano 2016, 10, 3747 3754.

16. Patrick, D. L.; Cee, V. J.; Beebe, T. P., "Molecule Corrals" for Studies of Monolayer Organic Films. Science 1994, 265, 231-234.

17. Patrick, D. L.; Cee, V. J.; Purcell, T. J.; Beebe, T. P., Defect Pinning in Monolayer Films by Highly Controlled Graphite Defects: Molecule Corrals. Langmuir 1996, 12, 1830-1835.

18. Patrick, D. L.; Cee, V. J.; Beebe, T. P., Mechanism of Molecular Ordering in Monolayer Liquid Crystal Films. The Journal of Physical Chemistry 1996, 100, 8478-8481.

19. Stevens, F.; Buehner, D.; Beebe, T. P., Ordering of Adsorbed Organic Monolayers Confined in Molecule Corrals during Scanning Tunneling Microscopy Observation. J. Phys. Chem. B 1997, 101, 6491-6496.

20. Kudernac, T.; Lei, S.; Elemans, J. A. A. W.; De Feyter, S., Two-dimensional supramolecular selfassembly: nanoporous networks on surfaces. Chem. Soc. Rev. 2009, 38, 402-421.

21. Lei, S.; Tahara, K.; De Schryver, F. C.; van der Auweraer, M.; Tobe, Y.; De Feyter, S., One Building Block, Two Different Supramolecular Surface-Confined Patterns: Concentration in Control at the Solid-Liquid Interface. Angew. Chem. Int. Ed. 2008, 47, 2964-2968.

22. Ahn, S.; Matzger, A. J., Six Different Assemblies from One Building Block: Two-Dimensional Crystallization of an Amide Amphiphile. J. Am. Chem. Soc. 2010, 132, 11364-11371.

23. Thi Ngoc Ha, N.; Gopakumar, T. G.; Hietschold, M., Polymorphism Driven by Concentration at the Solid-Liquid Interface. J. Phys. Chem. C 2011, 115, 21743-21749.

24. Blunt, M. O.; Adisoejoso, J.; Tahara, K.; Katayama, K.; Van der Auweraer, M.; Tobe, Y.; De Feyter, S., Temperature-Induced Structural Phase Transitions in a Two-Dimensional Self-Assembled Network. J. Am. Chem. Soc. 2013, 135, 12068-12075.

25. Gutzler, R.; Sirtl, T.; Dienstmaier, J. r. F.; Mahata, K.; Heckl, W. M.; Schmittel, M.; Lackinger, M., Reversible Phase Transitions in Self-Assembled Monolayers at the Liquid-Solid Interface: Temperature-Controlled Opening and Closing of Nanopores. J. Am. Chem. Soc. 2010, 132, 50845090.

26. Mazur, U.; Hipps, K. W., Kinetic and Thermodynamic Processes of Organic Species at the Solution-Solid Interface: The View through an STM. Chem. Commun. 2015, 51, 4737-4749. 
27. Bellec, A.; Arrigoni, C.; Schull, G.; Douillard, L.; Fiorini-Debuisschert, C.; Mathevet, F.; Kreher, D.; Attias, A.-J.; Charra, F., Solution-Growth Kinetics and Thermodynamics of Nanoporous SelfAssembled Molecular Monolayers. J. Chem. Phys. 2011, 134, 124702-7.

28. Kampschulte, L.; Lackinger, M.; Maier, A.-K.; Kishore, R. S. K.; Griessl, S.; Schmittel, M.; Heckl, W. M., Solvent Induced Polymorphism in Supramolecular 1,3,5-Benzenetribenzoic Acid Monolayers. J. Phys. Chem. B 2006, 110, 10829-10836.

29. Zhang, X.; Chen, T.; Chen, Q.; Deng, G.-J.; Fan, Q.-H.; Wan, L.-J., One Solvent Induces a Series of Structural Transitions in Monodendron Molecular Self-Assembly from Lamellar to Quadrangular to Hexagonal. Chem. Eur. J. 2009, 15, 9669-9673.

30. Yang, Y.; Wang, C., Solvent Effects on Two-Dimensional Molecular Self-Assemblies Investigated by using Scanning Tunneling Microscopy. Curr. Opin. Colloid Int. 2009, 14, 135-147.

31. Balandina, T.; Tahara, K.; Sändig, N.; Blunt, M. O.; Adisoejoso, J.; Lei, S.; Zerbetto, F.; Tobe, Y.; De Feyter, S., Role of Substrate in Directing the Self-Assembly of Multicomponent Supramolecular Networks at the Liquid-Solid Interface. ACS Nano 2012, 6, 8381-8389.

32. Katsonis, N.; Marchenko, A.; Fichou, D., Substrate-Induced Pairing in 2,3,6,7,10,11-Hexakisundecalkoxy-triphenylene Self-Assembled Monolayers on Au(111). J. Am. Chem. Soc. 2003, 125, 13682-13683.

33. Xie, Z. X.; Xu, X.; Mao, B. W.; Tanaka, K., Self-Assembled Binary Monolayers of n-Alkanes on Reconstructed $\mathrm{Au}(111)$ and HOPG Surfaces. Langmuir 2002, 18, 3113-3116.

34. Cometto, F. P.; Kern, K.; Lingenfelder, M., Local Conformational Switching of Supramolecular Networks at the Solid/Liquid Interface. ACS Nano 2015, 9, 5544-5550.

35. Lee, S.-L.; Fang, Y.; Velpula, G.; Cometto, F. P.; Lingenfelder, M.; Müllen, K.; Mali, K. S.; De Feyter, S., Reversible Local and Global Switching in Multicomponent Supramolecular Networks: Controlled Guest Release and Capture at the Solution/Solid Interface. ACS Nano 2015, 9, 1160811617.

36. Ubink, J.; Enache, M.; Stöhr, M., Bias-induced Conformational Switching of Supramolecular Networks of Trimesic Acid at the Solid-Liquid Interface. J. Chem. Phys. 2018, 148, 174703.

37. Li, S.-Y.; Yang, X.-Q.; Chen, T.; Wang, D.; Wang, S.-F.; Wan, L.-J., Tri-Stable Structural Switching in 2D Molecular Assembly at the Liquid/Solid Interface Triggered by External Electric Field. ACS Nano 2019, 13, 6751-6759.

38. MacLeod, J. M.; Lipton-Duffin, J. A.; Cui, D.; De Feyter, S.; Rosei, F., Substrate Effects in the Supramolecular Assembly of 1,3,5-Benzene Tricarboxylic Acid on Graphite and Graphene. Langmuir 2015, 31, 7016-7024.

39. Lee, S.-L.; Adisoejoso, J.; Fang, Y.; Tahara, K.; Tobe, Y.; Mali, K. S.; De Feyter, S., Efficient Screening of 2D Molecular Polymorphs at the Solution-Solid Interface. Nanoscale 2015, 7, 5344-5349. 
40. Mamdouh, W.; Uji-i, H.; Ladislaw, J. S.; Dulcey, A. E.; Percec, V.; De Schryver, F. C.; De Feyter, S., Solvent Controlled Self-Assembly at the Liquid-Solid Interface Revealed by STM.J. Am. Chem. Soc. 2005, 128, 317-325.

41. MacLeod, J. M.; Ivasenko, O.; Perepichka, D. F.; Rosei, F., Stabilization of Exotic Minority Phases in a Multicomponent Self-Assembled Molecular Network. Nanotechnology 2007, 18, 424031.

42. Nangia, A., Pseudopolymorph: Retain This Widely Accepted Term. Cryst. Growth Des. 2006, 6, 24.

43. Greenwood, J.; Phan, T. H.; Fujita, Y.; Li, Z.; Ivasenko, O.; Vanderlinden, W.; Van Gorp, H.; Frederickx, W.; Lu, G.; Tahara, K.; Tobe, Y.; Uji-i, H.; Mertens, S. F. L.; De Feyter, S., Covalent Modification of Graphene and Graphite Using Diazonium Chemistry: Tunable Grafting and Nanomanipulation. ACS Nano 2015, 9, 5520-5535.

44. Braganca, A. M.; Greenwood, J.; Ivasenko, O.; Phan, T. H.; Mullen, K.; De Feyter, S., The Impact of Grafted Surface Defects and their Controlled Removal on Supramolecular Self-Assembly. Chem. Sci. 2016, 7, 7028-7033.

45. Verstraete, L.; Greenwood, J.; Hirsch, B. E.; De Feyter, S., Self-Assembly under Confinement: Nanocorrals for Understanding Fundamentals of 2D Crystallization. ACS Nano 2016, 10, 1070610715.

46. Liu, G.-Y.; Xu, S.; Qian, Y., Nanofabrication of Self-Assembled Monolayers Using Scanning Probe Lithography. Acc. Chem. Res. 2000, 33, 457-466.

47. Garno, J. C.; Yang, Y.; Amro, N. A.; Cruchon-Dupeyrat, S.; Chen, S.; Liu, G.-Y., Precise Positioning of Nanoparticles on Surfaces Using Scanning Probe Lithography. Nano Lett. 2003, 3, 389-395.

48. Xu, S.; Liu, G.-y., Nanometer-Scale Fabrication by Simultaneous Nanoshaving and Molecular SelfAssembly. Langmuir 1997, 13, 127-129.

49. Tao, F.; Bernasek, S. L., Two-Dimensional Self-Assembly of a Two-Component Molecular System: Formation of an Ordered and Homogeneous Molecular Mesh.J. Am. Chem. Soc. 2005, 127, 1275012751.

50. De Feyter, S.; Gesquière, A.; Klapper, M.; Müllen, K.; De Schryver, F. C., Toward Two-Dimensional Supramolecular Control of Hydrogen-Bonded Arrays: The Case of Isophthalic Acids. Nano Lett. 2003, 3, 1485-1488.

51. Dickerson, P. N.; Hibberd, A. M.; Oncel, N.; Bernasek, S. L., Hydrogen-Bonding versus van der Waals Interactions in Self-Assembled Monolayers of Substituted Isophthalic Acids. Langmuir 2010, 26, 18155-18161.

52. Tao, F.; Bernasek, S. L., Self-assembly of 5-octadecyloxyisophthalic Acid and its Coadsorption with Terephthalic Acid. Surf. Sci. 2007, 601, 2284-2290.

53. Park, K.-W.; Adisoejoso, J.; Plas, J.; Hong, J.; Müllen, K.; De Feyter, S., Self-Assembly Behavior of Alkylated Isophthalic Acids Revisited: Concentration in Control and Guest-Induced Phase Transformation. Langmuir 2014, 30, 15206-15211. 
54. Taylor, C. R.; Day, G. M., Evaluating the Energetic Driving Force for Cocrystal Formation. Cryst. Growth Des. 2018, 18, 892-904.

55. Seibel, J.; Verstraete, L.; Hirsch, B. E.; Bragança, A. M.; De Feyter, S., Biasing Enantiomorph Formation via Geometric Confinement: Nanocorrals for Chiral Induction at the Liquid-Solid Interface. J. Am. Chem. Soc. 2018, 140, 11565-11568.

56. Fatima; Zepeda, M.; Oncel, N., Scanning Tunneling Microscopy/Spectroscopy Measurements and Density Functional Theory Calculations on Self-Assembled Monolayer of Octanoic Acid on Graphite. Thin Solid Films 2017, 623, 135-137.

57. Gutzler, R.; Lappe, S.; Mahata, K.; Schmittel, M.; Heckl, W. M.; Lackinger, M., Aromatic interaction vs.hydrogen bonding in self-assembly at the liquid-solid interface. Chem. Commun. 2009, 680682.

TOC graphic:
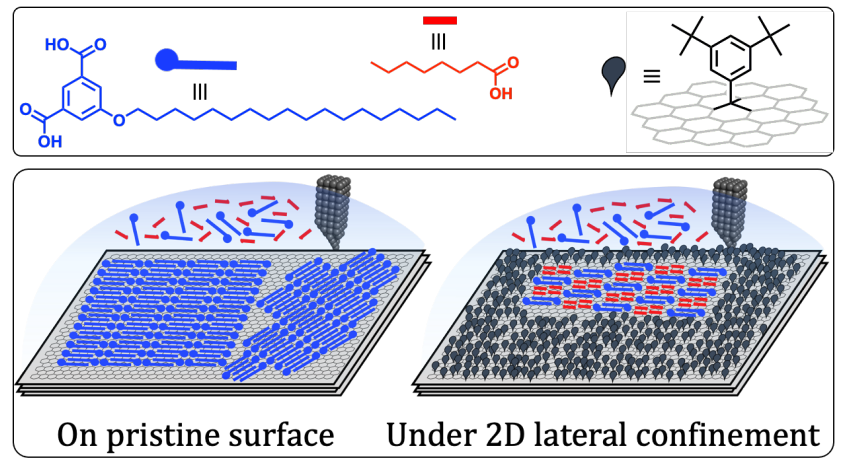